\title{
Robust Stabilization of Nonlinear Fractional Order Interconnected Systems Based on T-S Fuzzy Model
}

\author{
Yuting Li (10) \\ Xi'an Aeronautical University, Xi'an 710077, China \\ Correspondence should be addressed to Yuting Li; yutinglee@126.com
}

Received 10 December 2019; Revised 24 March 2020; Accepted 31 March 2020; Published 24 April 2020

Academic Editor: Átila Bueno

Copyright (c) 2020 Yuting Li. This is an open access article distributed under the Creative Commons Attribution License, which permits unrestricted use, distribution, and reproduction in any medium, provided the original work is properly cited.

This paper concerns robust stabilization of nonlinear fractional order interconnected systems. Based on uncertain fractional order Takagi-Sugeno fuzzy model and the fractional order extension of lyapunov direct method, a parallel distributed compensate controller is designed to asymptotically stabilize the fractional order interconnected systems. Then, a sufficient condition is given in the format of linear matrix inequalities. Simulation example is given to validate the effectiveness of the approach.

\section{Introduction}

Fractional order systems have attracted more and more attention due to its demonstrated application in many fields ([1-4]). Recently, a considerable literature has grown up around the theme of fractional order systems. For example, the problem of state estimation and synchronization for fractional order neural networks was discussed in [5-7]. Control problem for fractional order multiagent systems were introduced in [8-10]. Dynamic properties, control, and synchronization of fractional order chaotic systems were discussed in [11-14].

Large-scale interconnected system consists of a number of independent subsystems connected by some interconnections. Because interconnected systems are efficiently applied to practical systems such as economic systems, computer communication networks, and transportation systems, a considerable amount of literature has been published ([15-17]). Much of the previous research has focused on integer order. In 2013, a class of fractional order linear interconnected systems' stabilization problem was considered [18]. Then, the problem of robust resilient controllers synthesis for uncertain fractional order linear interconnected system was studied, and the state feedback nonfragile controller was designed under the additive and multiplicative gain perturbations [19]. Positive reduced- order functional observers for positive fractional order interconnected time-delay systems were designed by [20]. The problem of robust stabilization for positive fractional order interconnected systems with heterogeneous timevarying delays was proposed by [21]. The robust decentralized fault-tolerant resilient control for fractional order large-scale interconnected uncertain system was investigated in [22]. There are relatively few historical studies in the area of fractional order nonlinear interconnected systems.

Takagi-Sugeno (T-S) fuzzy model is one of the most common effective methods for approximating complex nonlinear systems. Over the past several years, there has been rapid development of interconnected systems by using T-S fuzzy model. For example, fuzzy large-scale interconnected systems were discussed in [23-25] and [26]. T-S fuzzy controllers for nonlinear multiple time-delay interconnected systems were studied in [27-29]. The finite-time stabilization problem for type-2 T-S interconnected nonlinear systems was investigated in $[30,31] . H_{\infty}$ control design for fuzzy discrete-time interconnected systems based on T-S fuzzy model was studied in [32-34]. Stability and stabilization problem for T-S Interconnected Fuzzy Systems by using different Lyapunov functions with slack variables are considered in [23]. The LMI stability conditions of fractional order uncertain T-S system were introduced in $[35,36]$. In this paper, we study the stability problem of 
nonlinear fractional order interconnected systems based on T-S fuzzy model and the fractional order extension of Lyapunov direct method.

This paper is organized as follows. Some preliminaries and the problem formulation are introduced first. The main results on the sufficient conditions of stabilization of nonlinear fractional order interconnected system are derived in Section 3. Section 4 interconnected fractional order chaotic systems to illustrate the effectiveness of the proposed results. Finally, the conclusions are drawn in Section 5.

Notations. The transpose of a matrix $A$ is denoted by $A^{T}$. $\operatorname{Sym}\{A\}$ is used to denote the expression $A^{T}+A$ and $*$ will be used in some expression to indicate a symmetric, i.e., $\left[\begin{array}{ll}X & Y \\ * & Z\end{array}\right]=\left[\begin{array}{cc}X & Y \\ Y^{T} & Z\end{array}\right]$.

\section{Preliminaries and Problem Formulation}

Consider a fractional order nonlinear interconnected system composed of $J$ fractional order subsystems $N_{j}, j=1, \ldots, J$. The $j$ th fractional order subsystem $N_{j}$ is described as follows:

$$
\begin{aligned}
D^{\alpha} x_{j}(t)= & f_{j}\left(x_{j}(t), u_{j}(t)\right)+\Delta f_{j}\left(x_{j}(t), u_{j}(t)\right) \\
& +\sum_{n=1, n \neq j}^{J} b_{n j}\left(x_{n}(t)\right)
\end{aligned}
$$

where $0<\alpha \leq 1$ is the fractional commensurate order, $f_{j}(\cdot)$ is the nonlinear vector-valued function, $\Delta f_{j}(\cdot)$ is the system uncertainties, $b_{n j}$ is the nonlinear interconnection between the $n$th and $j$ th subsystems, $x_{j}(t)$ is the state vector, and $u_{j}(t)$ is the input vector of the $j$ th fractional order subsystem, respectively. The operator $D^{\alpha}$ denotes ${ }_{t_{0}}^{C} D_{t}^{\alpha}$.

A set of fractional order T-S fuzzy model is employed here to deal with the control design problem of the fractional order nonlinear interconnected systems $N$. The $i$ th rule of the fuzzy model for the fractional order nonlinear interconnected subsystem $N_{j}$ is proposed as follows:

Plant Rule $i$ :

$$
\begin{aligned}
& \text { If } z_{1 j}(t) \text { is } M_{i 1 j} \text { and } \ldots \text { and } z_{p j}(t) \text { is } M_{i p j} \\
& \text { Then } \\
& D^{\alpha} x_{j}(t)=\left(A_{i j}+\Delta A_{i j}\right) x_{j}(t)+\sum_{n=1, n \neq j}^{J} \widehat{A}_{i n j} x_{n}(t)+ \\
& \left(B_{i j}+\Delta B_{i j}\right) u_{j}(t)
\end{aligned}
$$

where $i=1, \ldots, r_{j}, r_{j}$ is the number of IF-THEN rules, $M_{i h j}(h=1,2, \ldots, p)$ are the fuzzy sets, and $z_{1 j}(t), \ldots, z_{p j}(t)$ are the premise variables. $A_{i j}, \widehat{A}_{i n j}$, and $B_{i j}$ are constant matrices with appropriate dimension, while $\Delta A_{i j}$ and $\Delta B_{i j}$ are real-valued function matrices representing the time-varying parameter uncertainties that have the following form:

$$
\left[\Delta A_{i j} \Delta B_{i j}\right]=\left[D_{A i j} F_{A i j}(t) E_{A i j} D_{B i j} F_{B i j}(t) E_{B i j}\right],
$$

where $D_{A i j}, D_{B i j}, E_{A i j}, E_{B i j}$ are known constant matrices, and $F_{A i j}(t), F_{B i j}(t)$, are unknown matrices with Lebesgue measurable elements satisfying $F_{A i j}^{T}(t) F_{A i j}(t) \leq I$, $F_{B i j}^{T}(t) F_{B i j}(t) \leq I$. The final state of the fractional order fuzzy model is inferred as follows:

$$
\begin{aligned}
D^{\alpha} x_{j}(t)= & \sum_{i=1}^{r_{j}} h_{i j}(t)\left(A_{i j}+\Delta A_{i j}\right) x_{j}(t)+\sum_{n=1, n \neq j}^{J} \widehat{A}_{i n j} x_{n}(t) \\
& +\left(B_{i j}+\Delta B_{i j}\right) u_{j}(t),
\end{aligned}
$$

where $\quad h_{i j}(t)=w_{i j}(t) / \sum_{i=1}^{r_{j}} w_{i j}(t), \quad w_{i j}(t)=\prod_{q=1}^{p} M_{i q j}$ $\left(z_{q j}(t)\right), M_{i q j}\left(z_{q j}(t)\right)$ is the grade of the membership of $z_{q j}(t)$ in $M_{i q j}$. Notice the facts $w_{i j}(t) \geq 0$ for $i=1,2, \ldots, r_{j}$ and $\sum_{i=1}^{r_{j}} w_{i j}(t)>0$ for all $t$. Therefore, $h_{i j}(t) \geq 0$ for $i=1,2, \ldots, r_{j}$ and $\sum_{i=1}^{r_{j}} h_{i j}(t)=1$.

According to the decentralized fuzzy control scheme, a set of fuzzy controllers is synthesized via the parallel distributed compensation (PDC) to deal with the stabilization control for the fractional order nonlinear interconnected systems $N$. The $j$ th model-based fuzzy controller is

Control rule $i$ :

$$
\text { IF } z_{1 j}(t) \text { is } M_{i 1 j} \text { and } \ldots \text { and } z_{p j}(t) \text { is } M_{i p j} \text {, }
$$$$
\text { THEN } u_{j}(t)=K_{i j} x_{j}(t) \text {. }
$$

where $i=1,2, \ldots, r_{j}$.

Hence, the final output of the fuzzy controller has the form

$$
u_{j}(t)=\sum_{i=1}^{r_{j}} h_{i j}(t) K_{i j} x_{j}(t), \quad i=1,2, \ldots, r_{j}
$$

Substituting (4) into equation (3) yields the $j$ th closedloop subsystem as follows:

$$
\begin{aligned}
D^{\alpha} x_{j}(t)= & \sum_{i=1}^{r_{j}} \sum_{l=1}^{r_{j}} h_{i j}(t) h_{l j}(t) \\
& \cdot\left\{\left(\left(A_{i j}+\Delta A_{i j}\right)+\left(B_{i j}+\Delta B_{i j}\right) K_{l j}\right) x_{j}(t)\right. \\
& \left.+\sum_{n=1, n \neq j}^{J} \widehat{A}_{i n j} x_{n}(t)\right\} .
\end{aligned}
$$

Lemma 1 (see [37]). Let $x=0$ be an equilibrium point for the nonautonomous fractional order system $D^{\alpha} x(t)=$ $f(t, x)$. Let us assume that there exist a continuous Lyapunov function $V(x(t), t)$ and a scalar class-K function $\gamma_{1}(\cdot)$ such that $\forall x \neq 0$

$$
\begin{aligned}
\gamma_{1}(\|x(t)\|) & \leq V(x(t), t), \\
D^{\alpha} V(x(t), t) & \leq 0, \quad \text { with } \alpha \in(0,1],
\end{aligned}
$$

then the origin of the system is Lyapunov stable.

Lemma 2 (see [37]). Let $x(t) \in R^{n}$ be a vector of differentiable functions. Then, for any time instant $t \geq t_{0}$, the following relationship holds:

$$
\frac{1}{2} D^{\alpha} x^{T}(t) P x(t) \leq x^{T}(t) P D^{\alpha} x(t), \quad \forall \alpha \in(0,1], \forall t \geq t_{0},
$$


where $P \in R^{n \times n}$ is a constant, square, symmetric, and positive matrix.

Lemma 3 (see $[38,39])$. For any matrices $X$ and $Y$ with appropriate dimensions, we have $X^{T} Y+Y^{T} X \leq \sigma X^{T} X+$ $\sigma^{-1} Y^{T} Y$ for any $\sigma>0$.

Lemma 4 (see [40]). Given matrices $T, \Pi, N(t)$, and $M$ of appropriate dimensions and with $M$ symmetrical, then $M+$ $T N(t) \Pi+\Pi^{T} N^{T}(t) T^{T}<0$ holds for any $N(t)$ satisfying $N^{T}(t) N(t) \leq I$ if and only if there exists $\varepsilon>0$, such that $M+\varepsilon T T^{T}+\varepsilon^{-1} \Pi^{T} \Pi<0$.

Lemma 5 (Schur Complement), see [41]). For a given matrix $S=S^{T}$, the following assertions are equivalent:

$$
\begin{aligned}
& \text { (1) } S=\left[\begin{array}{ll}
S_{11} & S_{12} \\
S_{21} & S_{22}
\end{array}\right]<0 ; \\
& \text { (2) } S_{11}<0, S_{22}-S_{12}^{T} S_{11}^{-1} S_{12}<0 ; \\
& \text { (3) } S_{22}<0, S_{11}-S_{12} S_{22}^{-1} S_{12}^{T}<0 .
\end{aligned}
$$

\section{Main Results}

In this section, the stability of the fractional order nonlinear interconnected system $N$ is studied. A sufficient condition is established for system (5). Then the following theorem presents the main result.

Theorem 1. The closed-loop fractional order nonlinear interconnected system (5) is asymptotically stable if there are symmetric positive definite matrices $Q_{j}(j=1, \ldots, J)$, matrices $W_{i j}\left(i=1,2, \ldots, r_{j}\right)$, and real scalar constants $\varepsilon_{i j}, \eta_{i j}$, $\delta_{i j}, \mu_{l j}, \rho_{l j}$ and $\bar{\mu}$ such that

$$
\left[\begin{array}{cccc}
\Lambda_{i j} & Q_{j} E_{A i j}^{T} & W_{i j}^{T} E_{B i j}^{T} & Q_{j} \\
* & -\varepsilon_{i j} I & 0 & 0 \\
* & * & -\eta_{i j} I & 0 \\
& & & \\
* & * & * & -\bar{\mu}(J-1)^{-1} I
\end{array}\right]<0,
$$

where

$$
\begin{aligned}
\Lambda_{i j}= & \operatorname{Sym}\left\{A_{i j} Q_{j}+B_{i j} W_{i j}\right\}+\varepsilon_{i j} D_{A i j} D_{A i j}^{T}+\eta_{i j} D_{B i j} D_{B i j}^{T} \\
& +\sum_{n=1}^{J} \bar{\mu} \widehat{A}_{i n j} \widehat{A}_{i n j}^{T},
\end{aligned}
$$

$$
\left[\begin{array}{cccccc}
\Lambda_{i l j} & Q_{j} E_{A i j}^{T} & W_{l j}^{T} E_{B i j}^{T} & Q_{j} E_{A l j}^{T} & W_{i j}^{T} E_{B l j}^{T} & Q_{j} \\
* & -\gamma_{i j} I & 0 & 0 & 0 & 0 \\
* & * & -\delta_{i j} I & 0 & 0 & 0 \\
* & * & * & -\mu_{l j} I & 0 & 0 \\
* & * & * & * & -\rho_{l j} I & 0 \\
* & * & * & * & * & -\bar{\mu}(J-1)^{-1} I
\end{array}\right]<0,
$$

where

$$
\begin{aligned}
\Lambda_{i l j}= & \frac{1}{2} \operatorname{Sym}\left\{A_{i j} Q_{j}+B_{i j} W_{l j}+A_{l j} Q_{j}+B_{l j} W_{i j}\right\}+\gamma_{i j} D_{A i j} D_{A i j}^{T} \\
& +\delta_{i j} D_{B i j} D_{B i j}^{T}+\mu_{l j} D_{A l j} D_{A l j}^{T}+\rho_{l j} D_{B l j} D_{B l j}^{T}+\sum_{n=1}^{J} \bar{\mu} \widehat{A}_{i n j} \widehat{A}_{i n j}^{T} .
\end{aligned}
$$

The asymptotically stabilizing state feedback gain matrix is $K_{i j}=W_{i j} Q_{j}^{-1}$.

Proof. Let the Lyapunov function for the fractional order interconnected system $N$ be defined as

$$
V(t)=\sum_{j=1}^{J} v_{j}(t)=\sum_{j=1}^{J} 2 x_{j}^{T}(t) P_{j} x_{j}(t) .
$$

$P_{j}$ is real symmetric positive definite matrix. It follows from Lemma 1, the closed-loop fractional order nonlinear interconnected system (5) is asymptotically stable if $D^{\alpha} V(x(t), t) \leq 0$. Note that

$$
D^{\alpha} V(t)=\sum_{j=1}^{J} D^{\alpha} v_{j}(t)
$$

Applying Lemma 2 to $D^{\alpha} v_{j}(t)$, it can be obtained that $D^{\alpha} V(t) \leq \sum_{j=1}^{J}\left\{\left(D^{\alpha} x_{j}(t)\right)^{T} P_{j} x_{j}(t)+x_{j}^{T}(t) P_{j} D^{\alpha} x_{j}(t)\right\}$. 
Right side of inequality (15) can be represented by

$$
\begin{aligned}
& \sum_{j=1}^{J}\left\{x _ { j } ^ { T } ( t ) P _ { j } \sum _ { i = 1 } ^ { r _ { j } } \sum _ { l = 1 } ^ { r _ { j } } h _ { i j } ( t ) h _ { l j } ( t ) \left[\left(\left(A_{i j}+\Delta A_{i j}\right)+\left(B_{i j}+\Delta B_{i j}\right) K_{l j}\right) x_{j}(t)\right.\right. \\
& \left.\quad+\sum_{n=1, n \neq j}^{J} \widehat{A}_{i n j} x_{n}(t)\right]+\left[\sum_{i=1}^{r_{j}} \sum_{l=1}^{r_{j}} h_{i j}(t) h_{l j}(t)\right]\left[\left(\left(A_{i j}+\Delta A_{i j}\right)+\left(B_{i j}+\Delta B_{i j}\right)\right) K_{l j} x_{j}(t)\right. \\
& \left.\left.\quad+\sum_{n=1, n \neq j}^{J} \widehat{A}_{i n j} x_{n}(t)\right]^{T} P_{j} x_{j}\right\} \\
& =\sum_{j=1}^{J} \sum_{i=1}^{r_{j}} \sum_{l=1}^{r_{j}} h_{i j}(t) h_{l j}(t)\left\{x _ { j } ^ { T } ( t ) \left[\left(P_{j}\left(A_{i j}+\Delta A_{i j}\right)+\left(B_{i j}+\Delta B_{i j}\right) K_{l j}\right)\right.\right. \\
& \left.\left.\quad+\left(\left(A_{i j}+\Delta A_{i j}\right)+\left(B_{i j}+\Delta B_{i j}\right) K_{l j}\right)^{T} P_{j}\right] x_{j}(t)\right\} \\
& \quad+\sum_{j=1}^{J} \sum_{i=1}^{r_{j}} \sum_{l=1}^{r_{j}} \sum_{n=1, n \neq j}^{J} h_{i j}(t) h_{l j}(t)\left\{x_{j}^{T}(t) P_{j} \widehat{A}_{i n j} x_{n}+x_{n}^{T} \widehat{A}_{i n j}^{T} P_{j} x_{j}\right\} .
\end{aligned}
$$

By applying Lemma 3, it can be obtained

$$
\begin{aligned}
D^{\alpha} V(t) \leq & \sum_{j=1}^{J} \sum_{i=1}^{r_{j}} \sum_{l=1}^{r_{j}} h_{i j}(t) h_{l j}(t)\left\{x_{j}^{T}(t)\left[\operatorname{Sym}\left\{P_{j}\left(\left(A_{i j}+\Delta A_{i j}\right)+\left(B_{i j}+\Delta B_{i j}\right) K_{l j}\right)\right\}\right] x_{j}(t)\right\} \\
& +\sum_{j=1}^{J} \sum_{i=1}^{r_{j}} \sum_{n=1}^{J} h_{i j}(t)\left[\mu\left(\frac{J-1}{J}\right) x_{j}^{T}(t) x_{j}(t)+\mu^{-1}\left(x_{j}^{T}(t) P_{j} \widehat{A}_{i n j} \widehat{A}_{i n j}^{T} P_{j} x_{j}-\left(\frac{1}{J}\right) x_{j}^{T} P_{j} \widehat{A}_{i j j} \widehat{A}_{i j j}^{T} P_{j} x_{j}\right)\right] .
\end{aligned}
$$

In view of the matrix $\widehat{A}_{i j j}$ is equal to zero and $\sum_{l=1}^{r_{j}} h_{l j}=1$, we have

$$
\begin{aligned}
& \sum_{j=1}^{J} \sum_{i=1}^{r_{j}} \sum_{l=1}^{r_{j}} h_{i j}(t) h_{l j}(t) x_{j}^{T}(t)\left[\operatorname{Sym}\left\{P_{j}\left(\left(A_{i j}+\Delta A_{i j}\right)+\left(B_{i j}+\Delta B_{i j}\right) K_{l j}\right)\right\}+\right. \\
& \left.\sum_{n=1}^{J} \mu^{-1} P_{j} \widehat{A}_{i n j} \widehat{A}_{i n j}^{T} P_{j}+\mu(J-1) I\right] x_{j}(t) \\
& =\sum_{j=1}^{J} \sum_{i=1}^{r_{j}} h_{i j}^{2}(t) x_{j}^{T}(t)\left[\operatorname{Sym}\left\{P_{j}\left(\left(A_{i j}+\Delta A_{i j}\right)+\left(B_{i j}+\Delta B_{i j}\right) K_{i j}\right)\right\}+\right. \\
& \left.\sum_{n=1}^{J} \mu^{-1} P_{j} \widehat{A}_{i n j} \widehat{A}_{i n j}^{T} P_{j}+\mu(J-1) I\right] x_{j}(t)+2 \sum_{j=1}^{J} \sum_{i<l}^{r_{j}} h_{i j}(t) h_{l j}(t) x_{j}^{T}(t)\left[\frac{1}{2}\right. \\
& \cdot \operatorname{Sym}\left\{P_{j}\left(\left(A_{i j}+\Delta A_{i j}\right)+\left(B_{i j}+\Delta B_{i j}\right) K_{l j}+\left(A_{l j}+\Delta A_{l j}\right)+\left(B_{l j}+\Delta B_{l j}\right) K_{i j}\right)\right\} \\
& \left.\quad+\sum_{n=1}^{J} \mu^{-1} P_{j} \widehat{A}_{i n j} \widehat{A}_{i n j}^{T} P_{j}+\mu(J-1) I\right] x_{j}(t)<0 .
\end{aligned}
$$


If it is possible to assume each sum of (18) to be negative definite, respectively, then the fractional order nonlinear interconnected system is asymptotically stable.

First, assume that the first sum of the last equation in (18) is negative definite:

$$
\begin{array}{r}
\operatorname{Sym}\left\{P_{j}\left(\left(A_{i j}+\Delta A_{i j}\right)+\left(B_{i j}+\Delta B_{i j}\right) K_{i j}\right)\right\} \\
+\sum_{n=1}^{J} \mu^{-1} P_{j} \widehat{A}_{i n j} \widehat{A}_{i n j}^{T} P_{j}+\mu(J-1) I<0 .
\end{array}
$$

Equation (19) can be represented by

$$
\begin{aligned}
& \operatorname{Sym}\left\{P_{j}\left(A_{i j}+B_{i j} K_{i j}\right)\right\}+\operatorname{Sym}\left\{P_{j} D_{A i j} F_{A i j}(t) E_{A i j}\right\} \\
& \quad+\operatorname{Sym}\left\{P_{j} D_{B i j} F_{B i j}(t) E_{B i j} K_{i j}\right\}+\sum_{n=1}^{J} \mu^{-1} P_{j} \widehat{A}_{i n j} \widehat{A}_{i n j}^{T} P_{j} \\
& +\mu(J-1) I<0 .
\end{aligned}
$$

By applying Lemma 3 to (20), one obtains (20) holds if and only if there exist $\varepsilon_{i j}$ and $\eta_{i j}$ such that

$$
\begin{aligned}
\operatorname{Sym} & \left\{P_{j}\left(A_{i j}+B_{i j} K_{i j}\right)\right\}+\varepsilon_{i j} P_{j} D_{A i j} D_{A i j}^{T} P_{j}+\varepsilon_{i j}^{-1} E_{A i j}^{T} E_{A i j} \\
+ & \eta_{i j} P_{j} D_{B i j} D_{B i j}^{T} P_{j}+\eta_{i j}^{-1}\left(E_{B i j} K_{i j}\right)^{T} E_{B i j} K_{i j} \\
+ & \sum_{n=1}^{J} \mu^{-1} P_{j} \widehat{A}_{i n j} \widehat{A}_{i n j}^{T} P_{j}+\mu(J-1) I<0 .
\end{aligned}
$$

By the Schur complement, we can get

$$
\left.\begin{array}{cccc}
\Omega_{i j} & E_{A i j}^{T} & \left(E_{B i j} K_{i j}\right)^{T} & I \\
* & -\varepsilon_{i j} I & 0 & 0 \\
* & * & -\eta_{i j} I & 0 \\
* & * & * & -\mu^{-1}(J-1)^{-1} I
\end{array}\right]<0,
$$

where

$$
\begin{aligned}
\Omega_{i j}= & \operatorname{Sym}\left\{P_{j}\left(A_{i j}+B_{i j} K_{i j}\right)\right\}+\varepsilon_{i j} P_{j} D_{A i j} D_{A i j}^{T} P_{j} \\
& +\eta_{i j} P_{j} D_{B i j} D_{B i j}^{T} P_{j} \\
& +\sum_{n=1}^{J} \mu^{-1} P_{j} \widehat{A}_{i n j} \widehat{A}_{i n j}^{T} P_{j} .
\end{aligned}
$$

Define transformation matrix as $\operatorname{diag}\left[\begin{array}{llll}P_{j}^{-1} & I & I & I\end{array}\right]$ and take a congruence transformation to (22); this yields

$$
\left[\begin{array}{cccc}
P_{j}^{-1} \Omega_{i j} P_{j}^{-1} & P_{j}^{-1} E_{A i j}^{T} & P_{j}^{-1}\left(E_{B i j} K_{i j}\right)^{T} & P_{j}^{-1} I \\
* & -\varepsilon_{i j} I & 0 & 0 \\
* & * & -\eta_{i j} I & 0 \\
* & * & * & -\mu^{-1}(J-1)^{-1} I
\end{array}\right]<0 .
$$

Denoting $Q_{j}=P_{j}^{-1}, W_{i j}=K_{i j} P_{j}^{-1}$, and $\bar{\mu}=\mu^{-1}$, we have

$$
\begin{array}{cccc}
{\left[\begin{array}{cccc}
\Lambda_{i j} & Q_{j} E_{A i j}^{T} & W_{i j}^{T} E_{B i j}^{T} & Q_{j} \\
* & -\varepsilon_{i j} I & 0 & 0 \\
* & * & -\eta_{i j} I & 0 \\
* & * & * & -\bar{\mu}(J-1)^{-1} I
\end{array}\right]<0,} \\
\\
\quad j=1, \ldots, J, i=1, \ldots, r_{j},
\end{array}
$$

where

$$
\begin{aligned}
\Lambda_{i j}= & \operatorname{Sym}\left\{A_{i j} Q_{j}+B_{i j} W_{i j}\right\}+\varepsilon_{i j} D_{A i j} D_{A i j}^{T}+\eta_{i j} D_{B i j} D_{B i j}^{T} \\
& +\sum_{n=1}^{J} \bar{\mu} \widehat{A}_{i n j} \widehat{A}_{i n j}^{T} .
\end{aligned}
$$

The second LMI (11) can be established through a similar procedure. Assume that the second sum of the last equation in (18) is negative definite:

$$
\begin{aligned}
& \frac{1}{2} \operatorname{Sym}\left\{P _ { j } \left(\left(A_{i j}+\Delta A_{i j}\right)+\left(B_{i j}+\Delta B_{i j}\right) K_{l j}+\left(A_{l j}+\Delta A_{l j}\right)\right.\right. \\
& \left.\left.+\left(B_{l j}+\Delta B_{l j}\right) K_{i j}\right)\right\} \\
& +\sum_{n=1}^{J} \mu^{-1} P_{j} \widehat{A}_{i n j} \widehat{A}_{i n j}^{T} P_{j}+\mu(J-1) I<0 .
\end{aligned}
$$

Equation (27) can be represented by

$$
\begin{aligned}
& \frac{1}{2} \operatorname{Sym}\left\{P_{j}\left(A_{i j}+B_{i j} K_{l j}+A_{l j}+B_{l j} K_{i j}\right)\right\} \\
& +\operatorname{Sym}\left\{P_{j} D_{A i j} \frac{F_{A i j}(t)}{2} E_{A i j}\right\} \\
& +\operatorname{Sym}\left\{P_{j} D_{B i j} \frac{F_{B i j}(t)}{2} E_{B i j} K_{l j}\right\} \\
& +\operatorname{Sym}\left\{P_{j} D_{A l j} \frac{F_{A l j}(t)}{2} E_{A l j}\right\} \\
& +\operatorname{Sym}\left\{P_{j} D_{B l j} \frac{F_{B l j}(t)}{2} E_{B l j} K_{i j}\right\}+\sum_{n=1}^{J} \mu^{-1} P_{j} \widehat{A}_{i n j} \widehat{A}_{i n j}^{T} P_{j} \\
& +\mu(J-1) I<0 .
\end{aligned}
$$

By applying Lemma 3 to (28), one obtains (28) holds if and only if there exist $\gamma_{i j}, \delta_{i j}, \rho_{i j}$ and $\mu_{i j}$, such that 


$$
\begin{aligned}
& \frac{1}{2} \operatorname{Sym}\left\{P_{j}\left(A_{i j}+B_{i j} K_{l j}+A_{l j}+B_{l j} K_{i j}\right)\right\}+\gamma_{i j} P_{j} D_{A i j} D_{A i j}^{T} P_{j}+\gamma_{i j}^{-1} E_{A i j}^{T} E_{A i j} \\
& \quad+\delta_{i j} P_{j} D_{B i j} D_{B i j}^{T} P_{j}+\delta_{i j}^{-1}\left(E_{B i j} K_{l j}\right)^{T} E_{B i j} K_{l j}+\mu_{l j} P_{j} D_{A l j} D_{A l j}^{T} P_{j} \\
& +\mu_{l j}^{-1} E_{A l j}^{T} E_{A l j}+\rho_{l j} P_{j} D_{B l j} D_{B l j}^{T} P_{j}+\rho_{l j}^{-1}\left(E_{B l j} K_{i j}\right)^{T} E_{B l j} K_{i j} \\
& +\sum_{n=1}^{J} \mu^{-1} P_{j} \widehat{A}_{i n j} \widehat{A}_{i n j}^{T} P_{j}+\mu(J-1) I<0 .
\end{aligned}
$$

By the Schur complement, we can get

$$
\left[\begin{array}{cccccc}
\Omega_{i l j} & E_{A i j}^{T} & \left(E_{B i j} K_{l j}\right)^{T} & E_{A l j}^{T} & \left(E_{B l j} K_{i j}\right)^{T} & I \\
* & -\gamma_{i j} I & 0 & 0 & 0 & 0 \\
* & * & -\delta_{i j} I & 0 & 0 & 0 \\
* & * & * & -\mu_{l j} I & 0 & 0 \\
* & * & * & * & -\rho_{l j} I & 0 \\
* & * & * & * & * & -\mu^{-1}(J-1)^{-1} I
\end{array}\right]<0
$$

where

$$
\begin{aligned}
\Omega_{i l j}= & \frac{1}{2} \operatorname{Sym}\left\{P_{j}\left(A_{i j}+B_{i j} K_{l j}+A_{l j}+B_{l j} K_{i j}\right)\right\}+\gamma_{i j} P_{j} D_{A i j} D_{A i j}^{T} P_{j} \\
& +\delta_{i j} P_{j} D_{B i j} D_{B i j}^{T} P_{j}+\mu_{l j} P_{j} D_{A l j} D_{A l j}^{T} P_{j}+\rho_{l j} P_{j} D_{B l j} D_{B l j}^{T} P_{j} \\
& +\sum_{n=1}^{J} \mu^{-1} P_{j} \widehat{A}_{i n j} \widehat{A}_{i n j}^{T} P_{j} .
\end{aligned}
$$

Define transformation matrix as $\operatorname{diag}\left[\begin{array}{llllll}P_{j}^{-1} & I & I & I & I & I\end{array}\right]$ and take a congruence transformation to (30); this yields

$$
\left[\begin{array}{cccccc}
P_{j}^{-1} \Omega_{i l j} P_{j}^{-1} & P_{j}^{-1} E_{A i j}^{T} & P_{j}^{-1}\left(E_{B i j} K_{l j}\right)^{T} & P_{j}^{-1} E_{A l j}^{T} & P_{j}^{-1}\left(E_{B l j} K_{i j}\right)^{T} & P_{j}^{-1} I \\
* & -\gamma_{i j} I & 0 & 0 & 0 & 0 \\
* & * & -\delta_{i j} I & 0 & 0 & 0 \\
* & * & * & -\mu_{l j} I & 0 & 0 \\
* & * & * & * & -\rho_{l j} I & 0 \\
* & * & * & * & * & -\bar{\mu}(J-1)^{-1} I
\end{array}\right]<0,
$$


we have

$$
\left[\begin{array}{cccccc}
\Lambda_{i l j} & Q_{j} E_{A i j}^{T} & W_{l j}^{T} E_{B i j}^{T} & Q_{j} E_{A l j}^{T} & W_{i j}^{T} E_{B l j}^{T} & Q_{j} \\
* & -\gamma_{i j} I & 0 & 0 & 0 & 0 \\
* & * & -\delta_{i j} I & 0 & 0 & 0 \\
* & * & * & -\mu_{l j} I & 0 & 0 \\
* & * & * & * & -\rho_{l j} I & 0 \\
* & * & * & * & * & -\bar{\mu}(J-1)^{-1} I
\end{array}\right]<0,
$$

where

$$
\begin{aligned}
\Lambda_{i l j}= & \frac{1}{2} \operatorname{Sym}\left\{A_{i j} Q_{j}+B_{i j} W_{l j}+A_{l j} Q_{j}+B_{l j} W_{i j}\right\} \\
& +\gamma_{i j} D_{A i j} D_{A i j}^{T} \\
& +\delta_{i j} D_{B i j} D_{B i j}^{T}+\mu_{l j} D_{A l j} D_{A l j}^{T}+\rho_{l j} D_{B l j} D_{B l j}^{T} \\
& +\sum_{n=1}^{J} \bar{\mu} \widehat{A}_{i n j} \widehat{A}_{i n j}^{T} .
\end{aligned}
$$

This completes the proof.

When $\Delta A_{i j}$ and $\Delta B_{i j}$ are 0 , it is easy to get the following Corollary.

Corollary 1. The closed-loop fractional order nonlinear interconnected system (5) is asymptotically stable if there are symmetric positive definite matrices $Q_{j}(j=1, \ldots, J)$, matrices $W_{i j}\left(i=1,2, \ldots, r_{j}\right)$, and a real scalar constant $\bar{\mu}$ such that

$$
\left[\begin{array}{cc}
\Lambda_{i j} & Q_{j} \\
* & -\bar{\mu}(J-1)^{-1} I
\end{array}\right]<0, \quad i=1, \ldots, r_{j}, j=1, \ldots, J,
$$

with

$$
\begin{array}{r}
\Lambda_{i j}=\operatorname{Sym}\left\{A_{i j} Q_{j}+B_{i j} W_{i j}\right\}+\sum_{n=1}^{J} \bar{\mu} \widehat{A}_{i n j} \widehat{A}_{i n j}^{T}, \\
{\left[\begin{array}{cc}
\Lambda_{i l j} & Q_{j} \\
* & -\bar{\mu}(J-1)^{-1} I
\end{array}\right]<0, \quad 1 \leq i<l \leq r_{j},}
\end{array}
$$

with

$$
\begin{aligned}
\Lambda_{i l j}= & \frac{1}{2} \operatorname{Sym}\left\{A_{i j} Q_{j}+B_{i j} W_{l j}+A_{l j} Q_{j}+B_{l j} W_{i j}\right\} \\
& +\sum_{n=1}^{J} \bar{\mu} \widehat{A}_{i n j} \widehat{A}_{i n j}^{T} .
\end{aligned}
$$

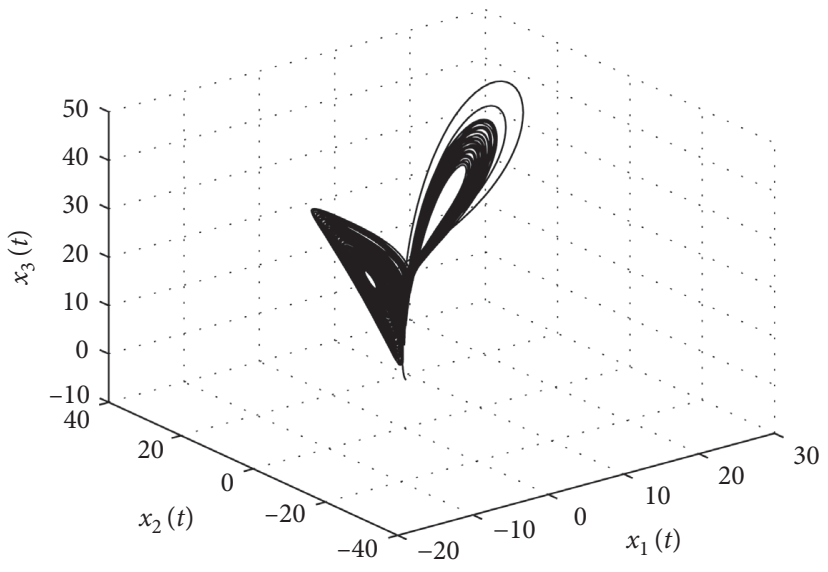

FIGURE 1: Chaotic behaviors of the fractional order uncertain Lorenz system.

The asymptotically stabilizing state feedback gain matrix is $K_{i j}=W_{i j} Q_{j}^{-1}$.

Remark 1. Since T-S fuzzy system can effectively approximate complex systems with nonlinearity, our model can be applied to a broad class of nonlinear fractional order interconnected systems. Most of all, stabilization of system can be developed by solving a set of LMIs. Moreover, when the number of the rules $r_{1}, \ldots, r_{j}$ is one, our model can be applied to solving fractional order linear interconnected system with uncertainties.

\section{Numerical Examples}

In this part, in order to show the effectiveness of the proposed method, a numerical example on interconnected fractional order chaotic systems will be provided.

Consider the asymptotical stability of nonlinear fractional order interconnected systems, and each subsystem is fractional order uncertain Lorenz chaotic system:

$$
\left\{\begin{array}{l}
D^{\alpha} x_{1}(t)=-(a+\Delta a) x_{1}(t)+(a+\Delta a) x_{2}(t) \\
D^{\alpha} x_{2}(t)=(c+\triangle c) x_{1}(t)-x_{2}(t)-x_{1}(t) x_{3}(t) \\
D^{\alpha} x_{3}(t)=x_{1}(t) x_{2}(t)-b x_{3}(t)
\end{array}\right.
$$

And when $a=10, \triangle a=\sin t, b=8 / 3, c=28, \triangle c=0.14$, and $\alpha=0.993$, chaotic behaviors of the fractional order uncertain Lorenz chaotic system are shown in Figure 1.

Let us consider two interconnected fractional order uncertain Lorenz chaotic system as follows: 


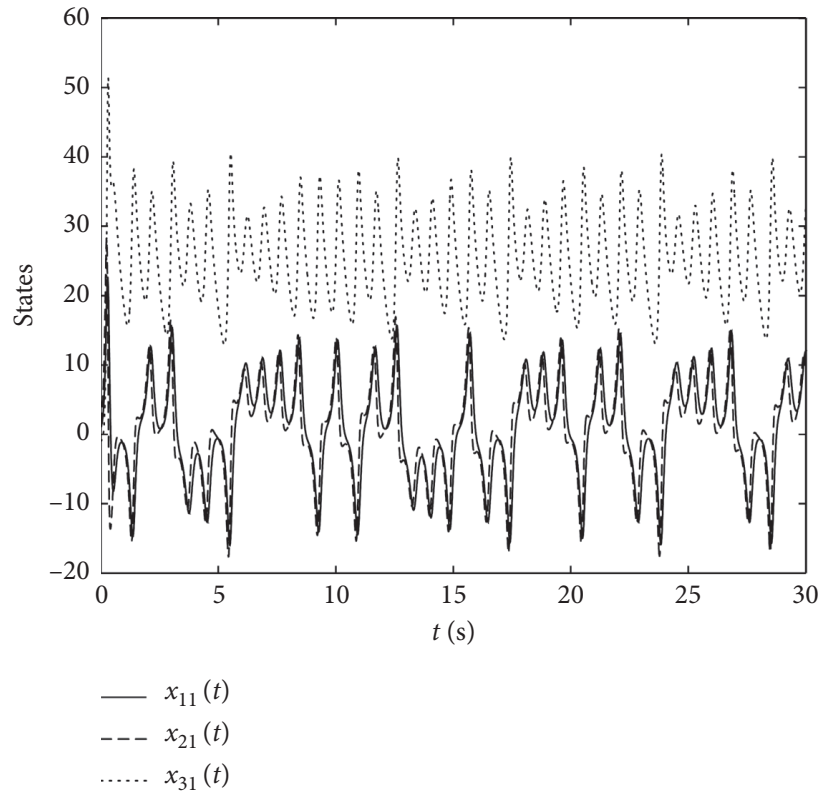

Figure 2: The state of subsystem 1 in nonlinear fractional order interconnected systems.

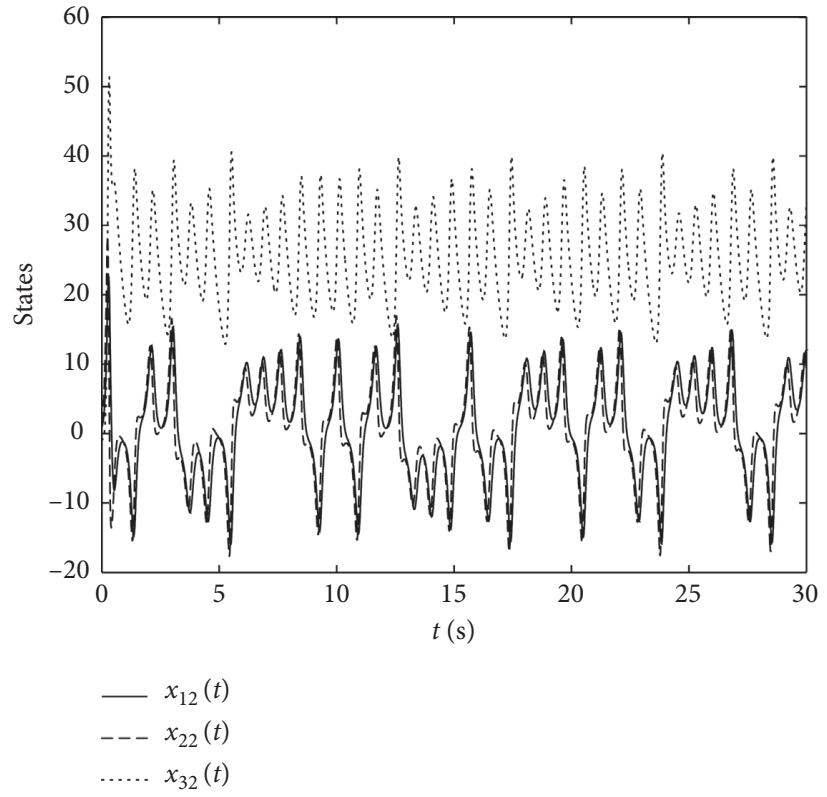

Figure 3: The state of subsystem 2 in nonlinear fractional order interconnected systems.

Subsystem 1:

$$
\left\{\begin{array}{l}
D^{\alpha} x_{11}(t)=-(a+\Delta a) x_{11}(t)+(a+\Delta a) x_{21}(t)+x_{12}(t)+x_{22}(t)+u_{1}(t), \\
D^{\alpha} x_{21}(t)=(c+\Delta c) x_{11}(t)-x_{21}(t)-x_{11}(t) x_{31}(t)+x_{12}(t)+x_{32}(t), \\
D^{\alpha} x_{31}(t)=x_{11}(t) x_{21}(t)-b x_{31}(t) .
\end{array}\right.
$$




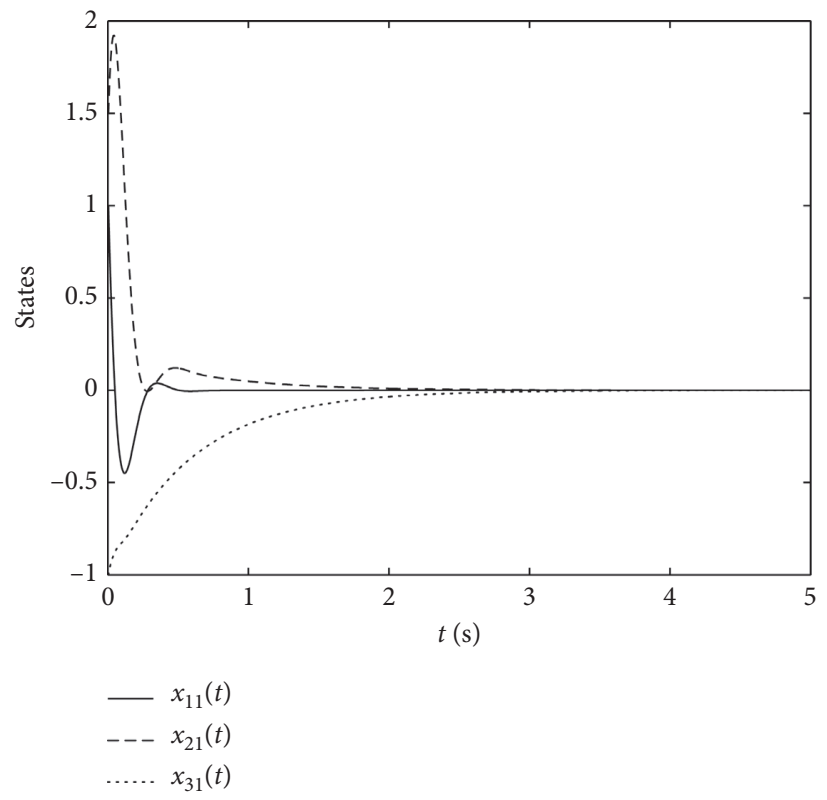

FIGURE 4: Control results of the fractional order nonlinear interconnected system.

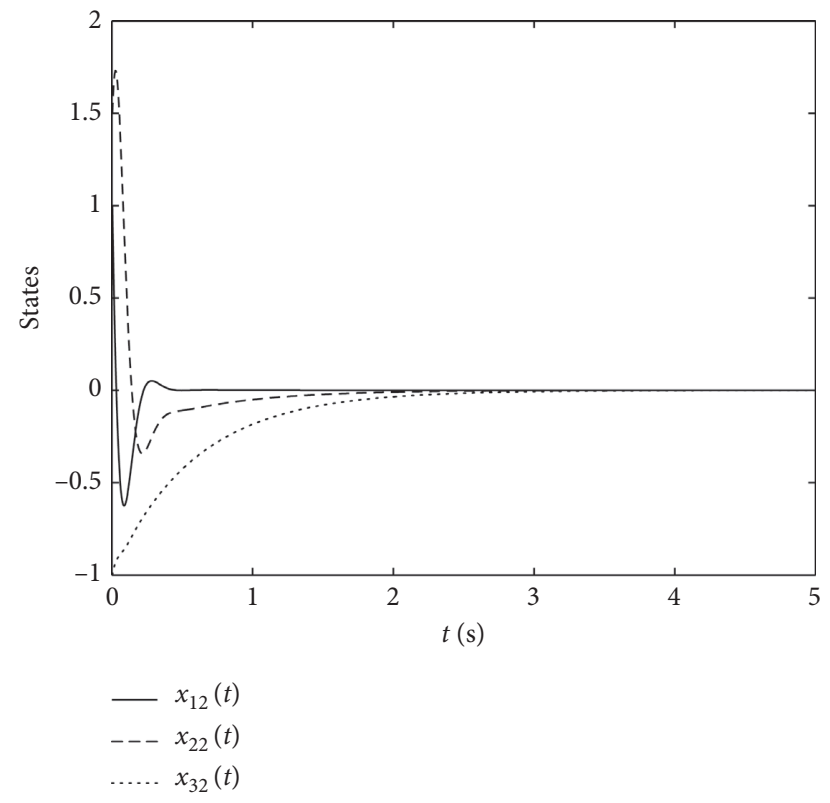

Figure 5: Control results of the fractional order nonlinear interconnected system.

Subsystem 2:

$$
\left\{\begin{array}{l}
D^{\alpha} x_{12}(t)=-(a+\triangle a) x_{12}(t)+(a+\triangle a) x_{22}(t)+x_{11}(t)+x_{21}(t)+u_{2}(t), \\
D^{\alpha} x_{22}(t)=(c+\triangle c) x_{12}(t)-x_{22}(t)-x_{12}(t) x_{32}(t)+x_{11}(t)+x_{31}(t), \\
D^{\alpha} x_{32}(t)=x_{12}(t) x_{22}(t)-b x_{32}(t) .
\end{array}\right.
$$


The state curves Nonlinear Fractional Order Interconnected Systems that without control, i.e., $u_{1} \equiv 0, u_{2} \equiv 0$ are shown in Figures 2 and 3.

Step 1. To stabilize the above fractional order interconnected system, we firstly establish fractional order T-S fuzzy model for each nonlinear fractional order subsystem. Assume that $x_{1 j}(t) \in[-d, d]$ and $d>0, d=30$, then we have

The fuzzy model of Subsystem $j$ :

Rule 1: IF $x_{1 j}(t)$ is $M_{1 j}$, THEN

$D^{\alpha} x_{j}(t)=\left(A_{1 j}+\triangle A_{1 j}\right) x_{j}(t)+\widehat{A}_{1 l j} x_{l}(t)+B_{1 j} u_{j}(t)$,

Rule 2: IF $x_{1 j}(t)$ is $M_{2 j}$, THEN

$D^{\alpha} x_{j}(t)=\left(A_{2 j}+\Delta A_{2 j}\right) x_{j}(t)+\widehat{A}_{2 l j} x_{l}(t)+B_{2 j} u_{j}(t)$.

where $x_{j}^{T}(t)=\left[x_{1 j}(t) x_{2 j}(t) x_{3 j}(t)\right], l \neq j$ and $j=1,2$.

$$
\begin{aligned}
A_{1 j} & =\left[\begin{array}{ccc}
-a & a & 0 \\
c & -1 & -d \\
0 & d & -b
\end{array}\right], \\
A_{2 j} & =\left[\begin{array}{ccc}
-a & a & 0 \\
c & -1 & d \\
0 & -d & -b
\end{array}\right], \\
\widehat{A}_{1 l j} & =\widehat{A}_{2 l j}=\left[\begin{array}{ccc}
1 & 1 & 0 \\
1 & 0 & 1 \\
0 & 0 & 0
\end{array}\right], \\
\Delta A_{1 j} & =\Delta A_{2 j}=\left[\begin{array}{ccc}
-\Delta a & \Delta a & 0 \\
\Delta c & 0 & 0 \\
0 & 0 & 0
\end{array}\right], \\
B_{1 j} & =B_{2 j}=\left[\begin{array}{l}
1 \\
0 \\
0
\end{array}\right] .
\end{aligned}
$$

Here $\Delta A_{1 j}$ and $\Delta A_{2 j}$ can be represented by

$$
\left[\begin{array}{ccc}
-0.1 & 0 & 0 \\
0 & 0.005 & 0 \\
0 & 0 & 0
\end{array}\right]\left[\begin{array}{ccc}
\sin (t) & 0 & 0 \\
0 & 1 & 0 \\
0 & 0 & 1
\end{array}\right]\left[\begin{array}{ccc}
10 & -10 & 0 \\
28 & 0 & 0 \\
0 & 0 & 0
\end{array}\right] \text {, }
$$

then we have $D_{A_{1 j}}=D_{A_{2 j}}=\left[\begin{array}{ccc}-0.1 & 0 & 0 \\ 0 & 0.005 & 0 \\ 0 & 0 & 0\end{array}\right]$, $E_{A_{1 j}}=E_{A_{2 j}}=\left[\begin{array}{ccc}10 & -10 & 0 \\ 28 & 0 & 0 \\ 0 & 0 & 0\end{array}\right]$, and $D_{B_{1 j}}=D_{B_{2 j}}=\left[\begin{array}{lll}0 & 0 & 0 \\ 0 & 0 & 0 \\ 0 & 0 & 0\end{array}\right]$, $E_{B_{1 j}}=E_{B_{2 j}}=\left[\begin{array}{l}0 \\ 0 \\ 0\end{array}\right]$ and the membership functions for Rule 1 and Rule 2 are: $M_{1 j}\left(x_{1 j}(t)\right)=(1 / 2)\left(1+x_{1 j}(t) / d\right)$, $M_{2 j}\left(x_{1 j}(t)\right)=(1 / 2)\left(1-x_{1 j}(t) / d\right)$.

Fuzzy controller of Subsystem $j$ :

Rule 1: IF $x_{1 j}(t)$ is $M_{1 j}$, THEN $u_{j}(t)=K_{1 j} x_{j}(t)$,

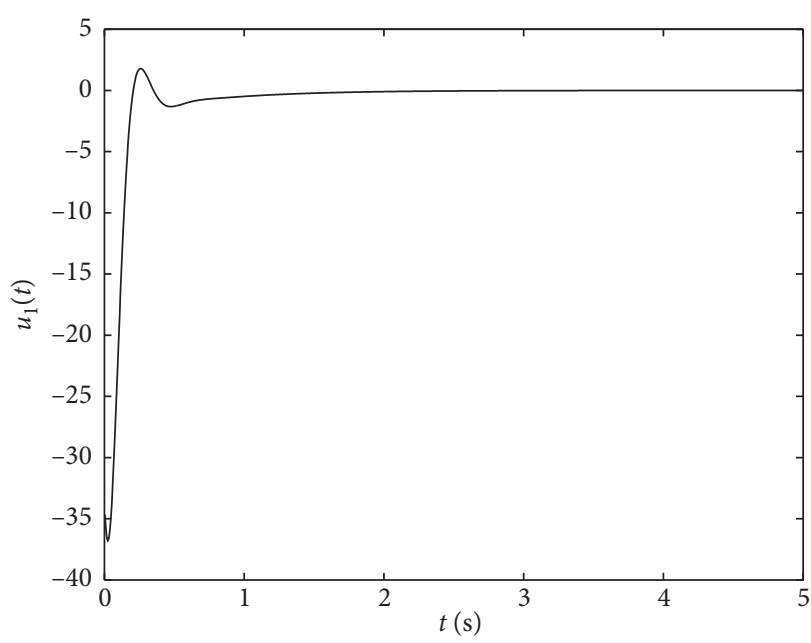

Figure 6: Control curve $u_{1}(t)$ of the fractional order interconnected system.

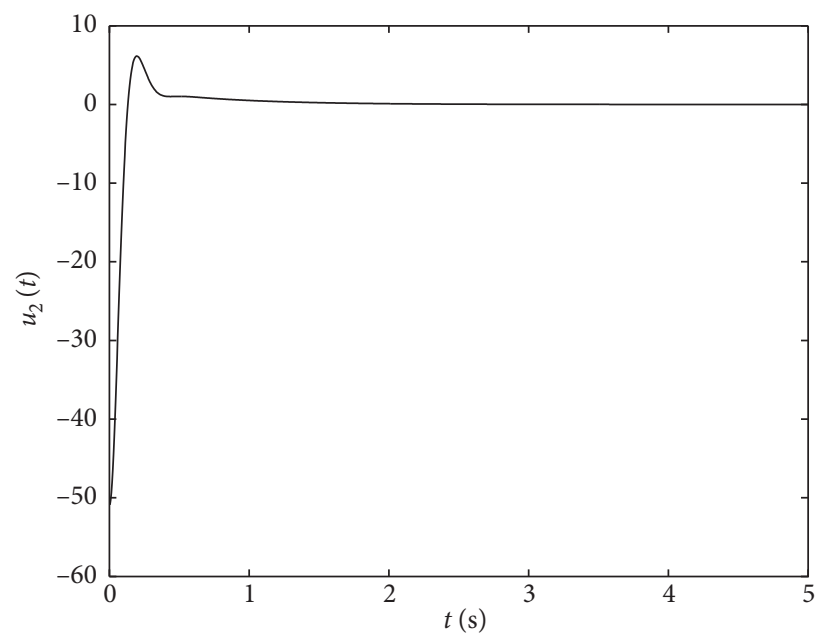

Figure 7: Control curve $u_{2}(t)$ of the fractional order interconnected system.

Rule 2: IF $x_{1 j}(t)$ is $M_{2 j}$, THEN $u_{j}(t)=K_{2 j} x_{j}(t)$.

The final output of the fuzzy controller is $u_{j}(t)=\sum_{i=1}^{2} h_{i j}(t) K_{i j} x_{j}(t)$.

Step 2. By applying Theorem 1 and using packages YALMIP in Matlab, we find the LMI (9) and (11) in Theorem 1 is feasible, a feasible solution is as follows:

$$
\begin{aligned}
& K_{11}=\left[\begin{array}{lll}
-7.4134 & -17.3762 & 3.7393
\end{array}\right] \\
& K_{21}=\left[\begin{array}{lll}
-9.6733 & -20.5994 & 0.8517
\end{array}\right] \\
& K_{12}=\left[\begin{array}{lll}
-10.7060 & -21.3431 & 5.6562
\end{array}\right] \\
& K_{22}=\left[\begin{array}{lll}
-13.8411 & -25.5120 & 1.4921
\end{array}\right]
\end{aligned}
$$

Therefore, the fractional order nonlinear fractional order interconnected system under fuzzy control law is determined to be asymptotically stable. The simulation results of each subsystem states under control are illustrated in 
Figures 4 and 5 shows that it is asymptotically stable, and the control curve of system is shown in Figures 6 and 7.

\section{Conclusion}

This paper focuses on the stability of the nonlinear fractional order interconnected systems. A useful stabilization approach has been given. The basis of this approach is to apply fractional order uncertain T-S fuzzy model to nonlinear fractional order interconnected systems. The PDC control design is carried out based on the fractional order T-S fuzzy model and the fractional order extension of Lyapunov direct method, a sufficient condition was given in terms of LMI. Finally, nonlinear fractional order interconnected systems was given to illustrate the effectiveness of the proposed theoretical results.

\section{Data Availability}

The data used to support the findings of this study are available from the corresponding author upon request.

\section{Conflicts of Interest}

The authors declare that they have no conflicts of interest.

\section{Acknowledgments}

This work was supported by Scientific Research Program Funded by Shaanxi Provincial Education Department (17JK0394) and Foundation of Xi'an Aeronautical University (2017ky0208).

\section{References}

[1] Y. Luo, Y. Q. Chen, L. Di, and H. Chao, "Lateral directional fractional order (PI $)^{\alpha}$ control of a small fixed-wing unmanned aerial vehicles: controller designs and flight tests," IET Control Theory \& Applications, vol. 5, no. 18, pp. 2156-2167, 2011.

[2] C. Riccardo, G. Dongola, L. Fortuna, and I. Petráś, Fractional Order Systems: Modeling and Control Applications, World Scientific, Singapore, 2010.

[3] V. K. Yadav, S. Das, B. S. Bhadauria, A. K. Singh, and M. Srivastava, "Stability analysis, chaos control of a fractional order chaotic chemical reactor system and its function projective synchronization with parametric uncertainties," Chinese Journal of Physics, vol. 55, no. 3, pp. 594-605, 2017.

[4] S. Ghasemi, A. Tabesh, and J. Askari-Marnani, "Application of fractional calculus theory to robust controller design for wind turbine generators," IEEE Transactions on Energy Conversion, vol. 29 , no. 3, pp. 780-787, 2014.

[5] B. Hu, Q. Song, and Z. Zhao, "Robust state estimation for fractional-order complex-valued delayed neural networks with interval parameter uncertainties: LMI approach," $A p$ plied Mathematics and Computation, vol. 373, Article ID 125033, 2020.

[6] H.-L. Li, L. Zhang, C. Hu, H. Jiang, and J. Cao, "Global mittagleffler synchronization of fractional-order delayed quaternion-valued neural networks: direct quaternion approach," Applied Mathematics and Computation, vol. 373, Article ID 125020, 2020.
[7] Y. Gu, H. Wang, and Y. Yu, "Synchronization for fractionalorder discrete-time neural networks with time delays," Applied Mathematics and Computation, vol. 372, Article ID 124995, 2020.

[8] E. Shahamatkhah and M. Tabatabaei, "Containment control of linear discrete-time fractional-order multi-agent systems with time-delays," Neurocomputing, vol. 385, pp. 42-47, 2020.

[9] X.-L. Yuan, L.-P. Mo, Y.-G. Yu, and G.-J. Ren, "Distributed containment control of fractional-order multi-agent systems with double-integrator and nonconvex control input constraints," International Journal of Control, Automation and Systems, pp. 195-209, 2020.

[10] Z. Yang, S. Zheng, F. Liu, and Y. Xie, "Adaptive output feedback control for fractional-order multi-agent systems," ISA Transactions, vol. 96, pp. 195-209, 2020.

[11] A. Buscarino, R. Caponetto, L. Fortuna, and E. Murgano, "Chaos in a fractional order duffing system: a circuit implementation," in Proceedings of the 2019 IEEE International Conference on Systems, Man and Cybernetics (SMC), pp. 2573-2577, IEEE, Bari, Italy, October 2019.

[12] Z. Li, D. Chen, J. Zhu, and Y. Liu, "Nonlinear dynamics of fractional order duffing system," Chaos, Solitons \& Fractals, vol. 81, pp. 111-116, 2015.

[13] J. Niu, R. Liu, Y. Shen, and S. Yang, "Chaos detection of duffing system with fractional-order derivative by Melnikov method," Chaos: An Interdisciplinary Journal of Nonlinear Science, vol. 29, no. 12, Article ID 123106, 2019.

[14] F. Meléndez-Vázquez and R. Martínez-Guerra, "Reducedorder fractional integral observer for synchronisation and anti-synchronisation of fractional-order chaotic systems," IET Control Theory \& Applications, vol. 12, no. 12, pp. 1755-1762, 2018.

[15] D. Zhai, X. Liu, and Y.-J. Liu, “Adaptive decentralized controller design for a class of switched interconnected nonlinear systems," IEEE Transactions on Cybernetics, vol. 50, no. 4, pp. 1644-1654, 2018.

[16] C. Hua, L. Zhang, and X. Guan, "Output feedback control for interconnected time-delay systems with prescribed performance," Neurocomputing, vol. 129, pp. 208-215, 2014.

[17] A. S. Tlili and N. Benhadj Braiek, " $H_{\infty}$ optimization-based decentralized control of linear interconnected systems with nonlinear interconnections," Journal of the Franklin Institute, vol. 351, no. 6, pp. 3286-3304, 2014.

[18] J. Li, J.-G. Lu, and Y. Chen, "Robust decentralized control of perturbed fractional-order linear interconnected systems," Computers \& Mathematics with Applications, vol. 66, no. 5, pp. 844-859, 2013.

[19] J. Lin, "Robust resilient controllers synthesis for uncertain fractional-order large-scale interconnected system," Journal of the Franklin Institute, vol. 351, no. 3, pp. 1630-1643, 2014.

[20] H. Trinh, D. C. Huong, and S. Nahavandi, "Observer design for positive fractional-order interconnected time-delay systems," Transactions of the Institute of Measurement and Control, vol. 41, no. 2, pp. 378-391, 2019.

[21] H. V. Le and K. T. Chu, "Robust control of positive fractionalorder interconnected systems with heterogeneous delays," Asian Journal of Control, vol. 21, no. 1, pp. 596-608, 2019.

[22] V. Nithya, R. Sakthivel, F. Alzahrani, and Y.-K. Ma, "Decentralized fault-tolerant resilient control for fractionalorder interconnected systems with input saturation," International Journal of Control, Automation and Systems, vol. 17, no. 11, pp. 2895-2905, 2019.

[23] A. Benzaouia, L. El Younsi, and A. El Hajjaji, "Stability and stabilization analysis for T-S interconnected fuzzy systems 
using LMIs," Circuits, Systems, and Signal Processing, vol. 38, no. 10, pp. 4504-4525, 2019.

[24] V.-P. Vu and W.-J. Wang, "Robust observer synthesis for the uncertain large-scale T-S fuzzy system," IET Control Theory \& Applications, vol. 13, no. 1, pp. 134-145, 2019.

[25] W.-W. Lin, W.-J. Wang, and S.-H. Yang, "A novel stabilization criterion for large-scale T-S fuzzy systems," IEEE Transactions on Systems Man and Cybernetics Part B-Cybernetics, vol. 37, pp. 1074-1079, 2007.

[26] C.-W. Chen, "Fuzzy control of interconnected structural systems using the fuzzy lyapunov method," Journal of $\mathrm{Vi}$ bration and Control, vol. 17, no. 11, pp. 1693-1702, 2011.

[27] F. H. Hsiao, C. W. Chen, Y. W. Liang, S. D. Xu, and W. L. Chiang, "T-S fuzzy controllers for nonlinear interconnected systems with multiple time delays," IEEE Transactions on Circuits and Systems I-Regular Papers, vol. 52, pp. 1883-1893, 2005.

[28] G. B. Koo, J. B. Park, and Y. H. Joo, "Robust fuzzy controller for large-scale nonlinear systems using decentralized static output-feedback," International Journal of Control, Automation and Systems, vol. 9, no. 4, pp. 649-658, 2011.

[29] F.-H. Hsiao, "Delay-dependent T-S fuzzy control for nonlinear interconnected systems: using dithers as auxiliaries," Applied Soft Computing, vol. 13, no. 11, pp. 4442-4459, 2013.

[30] Z. Wang, N. Rong, and H. Zhang, "Finite-time decentralized control of IT2 t-s fuzzy interconnected systems with discontinuous interconnections," IEEE Transactions on Cybernetics, vol. 49, no. 9, pp. 3547-3556, 2019.

[31] N. Rong, Z. Wang, and H. Zhang, "Finite-time stabilization for discontinuous interconnected delayed systems via interval type-2 t-s fuzzy model approach," IEEE Transactions on Fuzzy Systems, vol. 27, no. 2, pp. 249-261, 2018.

[32] L. E. Younsi, A. Benzaouia, and A. E. Hajjaji, "Decentralized control design for switching fuzzy large-scale t-s systems by switched lyapunov function with $h_{\infty}$ performance," International Journal of Fuzzy Systems, vol. 21, no. 4, pp. 11041116, 2019.

[33] Z. Zhong, S. Fu, T. Hayat, F. Alsaadi, and G. Sun, "Decentralized piecewise," Journal of the Franklin Institute, vol. 352, no. 9, pp. 3782-3807, 2015.

[34] H. Zhang, H. Zhong, and C. Dang, "Delay-dependent decentralized $h_{\infty}$ filtering for discrete-time nonlinear interconnected systems with time-varying delay based on the t-s fuzzy model," IEEE Transactions on Fuzzy Systems, vol. 20, no. 3, pp. 431-443, 2011.

[35] L. Junmin and L. Yuting, "Robust stability and stabilization of fractional order systems based on uncertain Takagi-Sugeno fuzzy model with the fractional order $1 \leq v<2$." Journal of Computational and Nonlinear Dynamics, vol. 8, no. 4, Article ID 041005, 2013.

[36] Y. Li and J. Li, "Stability analysis of fractional order systems based on T-S fuzzy model with the fractional order $\alpha$ : $0<\alpha<1$." Nonlinear Dynamics, vol. 78, no. 4, pp. 2909-2919, 2014.

[37] M. A. Duarte-Mermoud, N. Aguila-Camacho, J. A. Gallegos, and R. Castro-Linares, "Using general quadratic lyapunov functions to prove lyapunov uniform stability for fractional order systems," Communications in Nonlinear Science and Numerical Simulation, vol. 22, pp. 650-659, 2015.

[38] X. Li and C. E. D. Souza, "Criteria for robust stability and stabilization of uncertain linear systems with state delay," Automatica, vol. 33, no. 9, pp. 1657-1662, 1997.
[39] K. Zhou and P. P. Khargonekar, "Robust stabilization of linear systems with nonrm-bounded time-varying uncertainty," Systems \& Control Letters, vol. 10, no. 1, pp. 17-20, 1988.

[40] L. H. Xie, "Output feedback $H_{\infty}$ control of systems with parameter uncertainty," International Journal of Control, vol. 63, no. 4, pp. 741-750, 1996.

[41] S. Boyd, L. El Ghaoui, E. Feron, and V. Balakrishnan, "Linear matrix inequalities in system and control theory," in SIAM Studies in Applied Mathematics, University City Science Center, Philadelphia, PA, USA, 1994. 